\title{
Deactivation of poly ( 0 -aminophenol) film electrodes by storage without use in the supporting electrolyte solution and its comparison with other deactivation processes
}

\author{
R. Tucceri* \\ Instituto de Investigaciones Fisicoquímicas Teóricas y Aplicadas (INIFTA), CONICET, Facultad de Ciencias Exactas, Universidad Nacional de La Plata, Sucursal 4, Casilla de Correo \\ 16, 1900 La Plata, Argentina
}

\section{A R T I C L E I N F O}

\section{Article history:}

Received 6 July 2014

Received in revised form 22 October 2014

Accepted 8 December 2014

Available online 17 December 2014

\section{Keywords}

Poly(o-aminophenol)

Deactivation

Storage time

Electron diffusion coefficient

Redox site distribution

\begin{abstract}
A B S T R A C T
The effect of storage time without use (STWU) in the supporting electrolyte solution on the conducting properties of poly (o-aminophenol) (POAP) film electrodes was studied. Cyclic voltammetry (CV), rotating disc electrode voltammetry (RDEV), and surface resistance (SR) were employed. The storage of a POAP film without use for time periods longer than $32 \mathrm{~h}$ strongly reduces its electroactivity. Here, this effect is called deactivation. The attenuation of the voltammetric response of the polymer film with the increase of the storage time allows one to define a degree of deactivation $\left(\theta_{\mathrm{d}}\right)$. A decrease of the electron transport rate $\left(D_{\mathrm{e}}\right)$ with the increase of the degree of deactivation of POAP films was obtained from RDEV measurements. This effect was attributed to the increase of the mean distance between active redox sites in the bulk of the POAP film. The relative surface resistance change $(\Delta R / R)$ of a gold film coated with POAP is also attenuated by the increase of the STWU. The attenuation was attributed to interfacial (gold/POAP) redox site distributions where the nearest neighbor distance between redox sites gradually increases as the degree of deactivation increases. The deactivation of POAP films by the STWU studied in this work was compared with deactivations caused by other electrochemical and chemical treatments, such as prolonged potential cycling (PPC), high positive potential limits (HPPL) and soaking in a ferric ion solution (SFeIS), described in previous work.
\end{abstract}

(c) 2014 Elsevier B.V. All rights reserved.

\section{Introduction}

The oxidation of $o$-aminophenol (o-AP) on different electrode materials (gold, platinum, carbon, indium-tin oxide, etc.) in aqueous medium was shown to form poly-o-aminophenol (POAP) [1-3]. O-AP can be polymerized electrochemically in acidic, neutral and alkaline solutions. While a conducting film is only formed in acidic media, POAP synthesized in neutral and alkaline media leads to a nonconducting film [4]. The charge-transport process at POAP films synthesized in acid medium was mainly studied from the basic research viewpoint $[1,3,5,6]$. POAP synthesized in acidic medium is also found to be a useful material to build electrochemical sensors and electrocatalysts. The development of nitric oxide [7] and ferric cation [8] sensors based on POAP has been reported. POAP is also employed as conductive support of enzymes in biosensors [9] and as component of electrocatalysts for the oxidation of methanol [10].

\footnotetext{
* Fax: +54 (0221) 4254642.

E-mail address: rtucce@inifta.unlp.edu.ar
}

It is often assumed that after the different electrochemical and chemical treatments, at which POAP films synthesized in acid medium are subjected to both applied and basic studies, electron and ion conduction remains substantially unchanged. We have demonstrated that some of these treatments cause a decrease in the charge-transport rate of the polymer. For instance, in studies about electroactivity, POAP films are considered to be stable under extensive repetitive potential cycling in solvent-supporting electrolyte solution at sweep rates between 1 and $10^{5} \mathrm{mV} \mathrm{s}^{-1}$ [2]. We have observed a decrease in conductivity when POAP films in contact with the supporting electrolyte are subjected to a number of potential cycles beyond 500 at a scan rate of $0.010 \mathrm{~V} \mathrm{~s}^{-1}$ [11]. Furthermore, some applications of sensors and electrocatalysts based on POAP require positive potential limits beyond the potential range of maximal electroactivity of the polymer. In this regard, relations between the voltammetric peak current and analyte concentration in some sensors based on POAP [7] and also the dependence of the voltammetric peak current on polymer thickness in electrocatalysts where POAP is a conductive support [10] are obtained at positive potential values higher than $0.5 \mathrm{~V}$ (versus 
SCE). We have demonstrated in previous work that the application of positive potential limits beyond $0.5 \mathrm{~V}$ (SCE) leads to a decrease in POAP conductivity [12]. Other applications of POAP require the incorporation of metal ions into the polymer matrix. The interest in metal ion-POAP complexes arises from observations about their applications as sensors of metal ions in solution [8]. In this regard, it was demonstrated that POAP is able to capture Fe(III) ions from solution, which are partially reduced to Fe(II) inside the film, and then, the electrode exhibits a rapid (less than $10 \mathrm{~s}$ ) Nernstian response to $\mathrm{Fe}(\mathrm{III})$ ions in solution. In this application of POAP it is assumed that the polymer is electrically conductive and then, the electron exchange between the $\mathrm{Fe}(\mathrm{II})$ in the film and dissolved $\mathrm{Fe}(\mathrm{III})$ takes place rapidly at the polymer-solution interface. We have observed that the incorporation of $\mathrm{Fe}(\mathrm{III})$ ions into POAP leads to a decrease in the electron-transfer and ion-transfer rates across the POAP interfaces $[13,14]$.

In order to complete our previous studies about the limits of stability of POAP, a careful investigation has been carried out in the present work where it is demonstrated, employing CV, RDEV and the SR technique, that POAP films also deactivate with the storage time without use (STWU) in the supporting electrolyte. In this respect, it is expected to gain a further insight into the durability limits of the polymer because while in some basic studies POAP films are considered to be stable for long time periods under storage without use in the supporting electrolyte solution [2], a $43 \%$ decrease in the response of a peroxide hydrogen biosensor based on POAP was observed after some days under storage in solution [9]. The effect of the STWU on the electron conduction of POAP studied in this work is also compared with the effects on the electron-transport rate caused by other electrochemical and chemical treatments described in previous work [11-14]. It should be kept in mind that in the present work, as in previous ones [11-14], we are only interested in the stability and durability limits of POAP in terms of the charge conduction process. It is not the aim of the present work to analyze the physicochemical phenomena involved in POAP deactivation. In this regard, further work is in progress to elucidate the chemical and physical transformations that lead to a decrease in POAP conductivity after PPC, application of HPPL, SFeIS, and also long storage time without use in the supporting electrolyte.

\section{Experimental}

\subsection{CV and RDEV experiments}

A gold rotating disc electrode (RDE) was used as base electrode to deposit POAP films in CV and RDEV experiments. This gold RDE consisted of a gold rod press-fitted with epoxy resin into a Teflon sleeve so as to leave a $1 \mathrm{~cm}^{2}$ disc area exposed. The electrode was carefully polished with emery paper of decreasing grit size followed by alumina suspensions of $1,0.3$ and $0.05 \mu \mathrm{m}$, respectively, until a mirror-like finish was obtained. Then, it was submitted to ultrasonic cleaning to remove residual abraded polishing material. In order to obtain a more specular gold surface to deposit POAP films, a gold film about $50 \mathrm{~nm}$ in thickness was deposited by vacuum evaporation ( $\sim 10^{-7}$ Torr) on the gold disc. Then, in all experiments carried out in this work, POAP films were deposited on a specular gold film surface. POAP films were grown on these base electrodes following the procedure described in $[1,2]$. That is, polymer films were obtained by immersing the base electrode in a $10^{-3} \mathrm{M}$ orthoaminophenol $+0.4 \mathrm{M} \mathrm{NaClO}_{4}+0.1 \mathrm{M} \mathrm{HClO}_{4}$ solution and cycling the potential between -0.25 and $0.8 \mathrm{~V}$ (versus SCE) at a scan rate $v=0.05 \mathrm{~V} \mathrm{~s}^{-1}$. In the same way as in [11], POAP films were grown up to an approximate thickness of $\phi_{\mathrm{p}} \sim 60 \mathrm{~nm}$ by using a reduction charge $\left(Q_{\text {Red, } \mathrm{T}}=2.8 \mathrm{mC} \mathrm{cm}^{-2}\right)$ versus the ellipsometric thickness working curve [15]. These POAP-coated gold film electrodes were then rinsed and transferred to the supporting electrolyte solution $\left(0.4 \mathrm{M} \mathrm{NaClO}_{4}+0.1 \mathrm{M} \mathrm{HClO}_{4}\right)$ free of monomer, where they were stabilized by a continuous potential cycling within the potential range $-0.2<E<0.5 \mathrm{~V}$ at a scan rate of $0.01 \mathrm{~V} \mathrm{~s}^{-1}$. POAP films were considered to be stabilized after 50 cycles. These POAP films are herein called nondeactivated films. A large-area gold grid was used as counterelectrode. All the potentials reported in this work are referred to the SCE.

A series of eight POAP-coated RDEs was prepared (see first column of Table 1) and each one of them was successively deactivated in an individual experiment. That is, each POAP film was stored without use in a deoxygenated supporting electrolyte solution for a different time period (see second column of Table 1) and then, it was cycled within the potential region $-0.2<E<0.5 \mathrm{~V}$ to obtain a stable voltammetric response (50 cycles at $0.01 \mathrm{~V} \mathrm{~s}^{-1}$ ). Then, the corresponding $j-E$ responses for each one of the eight POAP films were compared. An attenuation of the voltammetric response, with respect to that of a nondeactivated film, was observed for the stored films. These POAP films are herein called deactivated films. Then, with both nondeactivated and deactivated POAP films, RDEV experiments were performed in the presence of a solution containing equimolar concentrations of $p$-benzoquinone (Q) and hydroquinone (HQ) species $\left(0.1 \mathrm{M} \mathrm{HClO}_{4}+0.4 \mathrm{M}\right.$ $\left.\mathrm{NaClO}_{4}+2 \times 10^{-3} \mathrm{M} \mathrm{Q} / \mathrm{HQ}\right)$. Stationary current-potential curves $(I-E)$ at different electrode rotation rates, $\Omega$, were recorded. From these curves, cathodic and anodic limiting current versus electrode rotation rate $\left(I_{\lim }\right.$ versus $\left.\Omega^{1 / 2}\right)$ dependences were obtained. In some experiments the $\mathrm{HQ} / \mathrm{Q}$ redox couple concentration in solution was varied.

In CV and RDEV measurements a PAR Model 173 potentiostat and a PAR Model 175 function generator were used. An X1-X2-Y Hewlett-Packard Model 7046 B plotter was used to record $j-E$ and steady-state current-potential curves $I-E$. The electrode rotation speed, $\Omega$, was controlled with homemade equipment that allows one to select a constant $\Omega$ in the range $50 \mathrm{rev} \mathrm{\textrm {min } ^ { - 1 }}$ $<\Omega<8000 \mathrm{rev} \mathrm{min}^{-1}$. This was periodically controlled with a digital photo tachometer (Power Instruments Inc., model 891).

AR grade chemicals were used throughout. $O$-aminophenol (Fluka) was purified as described elsewhere [1,2]. $\mathrm{HClO}_{4}$ and $\mathrm{NaClO}_{4}$ (Merck) were used without further purification. Benzoquinone and hydroquinone (Merck) were also used without

Table 1

Effect of the storage time without use (STWU) in the supporting electrolyte solution on the voltammetric charge of a POAP film.

\begin{tabular}{llll}
\hline${ }^{\mathrm{a}}$ POAP films & ${ }^{\mathrm{b}}$ Storage time without use $(\mathrm{h})$ & ${ }^{\mathrm{c}} Q_{\text {Red,c }}\left(\mathrm{mC} \mathrm{cm}^{-2}\right)$ & ${ }^{\mathrm{d}} \theta_{\mathrm{c}}^{\mathrm{d}}$ \\
\hline 1 & 32 & 2.65 & 0.05 \\
2 & 45 & 2.52 & 0.10 \\
3 & 52 & 2.37 & 0.15 \\
4 & 60 & 2.10 & 0.25 \\
5 & 72 & 1.83 & 0.35 \\
6 & 83 & 1.67 & 0.41 \\
7 & 96 & 1.12 & 0.62 \\
8 & 120 & 0.62 & 0.77
\end{tabular}

a Numbers 1 to 8 represent different deactivated POAP films.

b Storage time without use (in hours) of each POAP film in the supporting electrolyte solution.

c Voltammetric reduction charge of the different deactivated POAP films after being stored without use in the supporting electrolyte solution for the time periods listed in column 2.

${ }^{d}$ Degree of deactivation of each one of the POAP films after being stored without use in the supporting electrolyte solution for the time periods listed in column 2 . The degree of deactivation achieved by each film after storage was calculated from $\theta_{\mathrm{d}}=1-\left(Q_{\text {Red,c }} / Q_{\text {Red,T }}\right)$, where $Q_{\text {Red,T }}\left(=2.8 \mathrm{mC} \mathrm{cm}^{-2}\right)$ is the voltammetric reduction charge of a nondeactivated film. 
purification. The solutions were prepared with water purified using a Millipore Milli-Q system.

\subsection{Surface resistance measurements}

The experimental arrangement employed to perform SR experiments is one in which a POAP film is supported on a rectangular thin gold film. Eight thin gold film electrodes of constant thickness $\phi_{\mathrm{m}} \sim 30 \mathrm{~nm}$ were prepared by vacuum evaporation. All these electrodes exhibit initial resistance $(R)$ values of about $20.02 \mathrm{ohm}$. The relationship between the length $l$ and the width $w(G=l / w)$ of these gold film electrodes was 2 . The electrode area was $1 \mathrm{~cm}^{2}$. POAP films of $60 \mathrm{~nm}$ were grown on these base electrodes following the procedure previously described. A series of eight POAP-coated gold film electrodes was prepared and each one of them was successively deactivated by storage in an individual experiment, as indicated previously employing the same time sequence listed in column 2 of Table 1 . Then, the corresponding $j-E$ and $\Delta R / R-E$ stabilized responses ( 50 cycles) for each one of the eight deactivated POAP films in the supporting electrolyte solution $(0.4 \mathrm{M}$ $\mathrm{NaClO}_{4}+0.1 \mathrm{M} \mathrm{HClO}_{4}$ ) were recorded. In some experiments the thickness of the POAP film was varied.

The experimental setup for simultaneous voltammetric and SR measurements on thin film electrodes has previously been described in detail [16]. The electrochemical cell was also the same as that described previously [17]. The electrode resistance change was measured employing the three-contact method described earlier [18]. In this method, the potential drop along the resistive electrode, together the polarization current, is measured as a function of the applied potential $E$. The voltage difference at the extremes of the film is directly proportional to the resistance, and thus to the resistivity of the electrode. In order to compare the $\Delta R / R-E$ responses of the POAP films deactivated by employing different procedures (STWU, HPPL and SFeIS), the voltage difference at the extremes of the gold films coated with POAP was always compensated at the negative potential value $E=-0.2 \mathrm{~V}$ (reduced state of POAP) and then, the $\Delta R / R-E$ records were started at $E=-0.2 \mathrm{~V}$ and going toward the positive potential direction up to $E=0.5 \mathrm{~V}$ (oxidized state of POAP) [11].

A PAR Model 173 potentiostat together with a PAR Model 175 function generator were also used for potentiodynamic measurements. The potential drop at the extremes of the film was measured with a Keithley Model 160 voltmeter.

\section{Results and discussion}

\subsection{Comparison of voltammetric responses of nondeactivated and deactivated POAP films}

The voltammetric response corresponding to a nondeactivated POAP film within the potential range comprised between $-0.2 \mathrm{~V}$ and $0.5 \mathrm{~V}$ is shown in Fig. 1(plot (a)). The POAP film maintains this response within the potential range $-0.2 \mathrm{~V}<E<0.5 \mathrm{~V}$ even after being stored without use in the supporting electrolyte solution for $31 \mathrm{~h}$. However, after a higher storage time, this response starts to change. Fig. 1 compares the stabilized $j-E$ responses of a nondeactivated POAP film (plot (a)) with those of the films (2), (3), (5), (7) and (8) (see first column of Table 1) that were stored without use in the supporting electrolyte for the time periods listed in column 2 of Table 1 . The more attenuated voltammetric response observed in Fig. 1 as the storage time increases indicates a deactivation of the POAP film. In this regard, voltammetric reduction charge values corresponding to the completely reduced POAP films were compared for a nondeactivated film $\left(Q_{\text {Red,T }}=2.8 \mathrm{mC} \mathrm{cm}^{-2}\right)$ and the

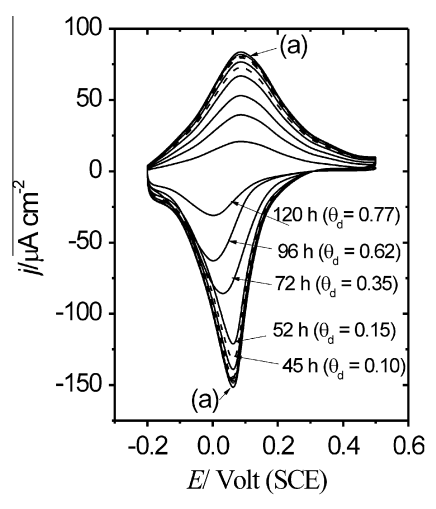

Fig. 1. Voltammetric $(j-E)$ responses for a $2.8 \mathrm{mC} \mathrm{cm}^{-2}\left(\phi_{\mathrm{p}}=60 \mathrm{~nm}\right)$ thick POAP film. (a) A nondeactivated POAP film $\left(\theta_{d}=0\right)$. The other voltammetric responses correspond to POAP films that were stored without use in the supporting electrolyte solution for the time periods (in hours) indicated in the figure. The degrees of deactivation of the films are indicated in Table 1. Electrolyte: $0.1 \mathrm{M} \mathrm{HClO}_{4}+0.4 \mathrm{M}$ $\mathrm{NaClO}_{4}$. Scan rate: $v=0.01 \mathrm{~V} \mathrm{~s}^{-1}$.

different deactivated films $\left(Q_{\text {Red,c }}\right)$ indicated in Table 1 . Then, a degree of deactivation (column 4 of Table 1 ) was defined as

$\theta_{\mathrm{d}}=1-\left(Q_{\text {Red,c }} / Q_{\text {Red,T }}\right)$

where $Q_{\text {Red,c }}$ is the total reduction charge assessed by integration of the corresponding $j-E$ response for a deactivated film, and $Q_{\text {Red,T }}=2.8 \mathrm{mC} \mathrm{cm}^{-2}$ is the total reduction charge for the nondeactivated film. Thus, for a nondeactivated POAP film (plot (a) in Fig. 1) the degree of deactivation was $\theta_{\mathrm{d}}=0$, taking $Q_{\mathrm{Red}, \mathrm{T}}=2.8 \mathrm{mC} \mathrm{cm}^{-2}$ as reference charge. However, values of $\theta_{\mathrm{d}}>0$ are indicative of POAP films that have been deactivated by storage in the supporting electrolyte solution. The number of exchanged electrons, $n$, during the switching redox of POAP has been obtained from optical measurements [5]. An $n$ value of around 0.4 was obtained for the positivegoing potential scan. Thus, it is considered that during the oxidative doping of POAP, one out of 4-5 amine sites (reduced state) undergoes oxidation to the corresponding imine site; then, we consider the cathodic charge $\left(Q_{\text {Red,c }}\right)$ as more representative of the electroactivity of POAP than the anodic one. In this connection, it can be seen from the voltammetric response of POAP shown in Fig. 1 that the cathodic peak is narrow, as compared with the anodic one. The asymmetry has been explained on the basis of interaction parameters. The interaction parameter for the anodic process of POAP is $r_{\mathrm{a}}=-0.55 \mathrm{M}^{-1}$ and that for the cathodic process is $r_{\mathrm{c}}=-0.13 \mathrm{M}^{-1}$ $[1,2]$. The fact that different values of $r_{\mathrm{a}}$ and $r_{\mathrm{c}}$ are obtained indicates a thermodynamic difference in the activity coefficients of the sites during oxidation and reduction processes. This disequilibrium effect has been observed in other films such as poly(vinylferrocene) [19]. The existence of "phase-like" behavior of the redox sites has been proposed to explain these effects on polymer films [20]. This implies that oxidized and reduced sites are segregated into microscopic domains differing both in solvent content and in average oxidation states dynamically growing and shrinking during the redox process. Also, a dimensional analysis shows that the interaction coefficient is equivalent to a molar volume, and several assumptions about its meaning can be considered. For instance, the formalism developed by Chidsey and Murray [21] considers $r$ as an interaction energy between active sites in the polymer matrix. As $r_{\mathrm{a}}$ is more negative than $r_{\mathrm{c}}$, this would mean a higher repulsive interaction between anodic sites as compared with the cathodic ones. Another formalism to explain the nonideal behavior of POAP is to assume that the active centers can have different values of $E^{\circ}$ (standard potential of the redox reaction). A given distribution of $E^{\circ} \mathrm{s}$ for the active centers around an average value is equivalent to the effect of active center concentration. Whatever the 
physicochemical nature of $r$ might be, it is related to the interaction between centers in highly concentrated media (the redox site concentration in POAP is around $4.5 \mathrm{M} \mathrm{[2])} \mathrm{and} \mathrm{it} \mathrm{is} \mathrm{reasonable} \mathrm{to}$ presume that the different interactions at the oxidized and reduced states of POAP are responsible for the asymmetric redox response of the polymer.

\subsection{Rotating disc electrode voltammetry measurements in the presence of a $\mathrm{HQ} / \mathrm{Q}$ solution}

In previous work [22] RDEV experiments at gold electrodes coated with POAP films were carried out to study the diffusion processes of benzoquinone $(Q)$ and hydroquinone (HQ) species through nondeactivated films. Diffusion-limited currents at $E<0.0 \mathrm{~V}$ for $Q$ reduction and at $E>0.8 \mathrm{~V}$ for $H Q$ oxidation were observed. While the anodic limiting current corresponds to the oxidation of $H Q$ species that penetrate through the polymer film to reach the metal surface, cathodic limiting currents for $Q$ reduction are related to a rapid electron-transfer mediation at the POAP|solution interface, which occurs without significant penetration of $Q$ into the polymer layer. As we are interested in the mediation reaction where redox species are only present in the solution phase but not inside the film, and they participate in the interfacial electron exchange with the polymer at the film-solution boundary, only the electrochemical behavior of nondeactivated and deactivated POAP films at negative potential values $(E<0.0 \mathrm{~V})$ was considered in the present work.

Fig. 2 shows ( $I-E)$ curves corresponding to the deactivated POAP film with $\theta_{\mathrm{d}}=0.62$. Both anodic ( $\left.I_{\lim , \mathrm{a}}\right)$ and cathodic $\left(I_{\lim , \mathrm{c}}\right)$ limiting currents, at each $\Omega$ value, for a deactivated POAP film are lower than those for a nondeactivated one [22]. $I_{\text {lim,c }}$ versus $\Omega^{1 / 2}$ dependences at potential values $E<0.0 \mathrm{~V}$ for both nondeactivated and deactivated POAP films are shown in Fig. 3. For a nondeactivated POAP film a linear $I_{\text {lim,c }}$ versus $\Omega^{1 / 2}$ dependence, which follows the Levich equation, is obtained within a wide range of $\Omega$ values (empty circles in Fig. 3). However, for POAP films that have been deactivated by storage without use for different time periods, after a certain $\Omega$ value, a constant cathodic limiting current value, $I_{\mathrm{lim}, \mathrm{c}}$, independent of $\Omega$ is achieved (black triangles in Fig. 3). Also, it is observed that the transition at which the cathodic limiting current becomes independent of $\Omega$ occurs at lower $\Omega$ values as the degree of deactivation increases.

Steady-state current-potential curves were interpreted in this work on the basis of the traditional electron hopping model [23-25]. In this regard, limiting current values at which $I_{\lim , c}\left(=I_{e}\right)$ becomes constant were considered as a representation of the

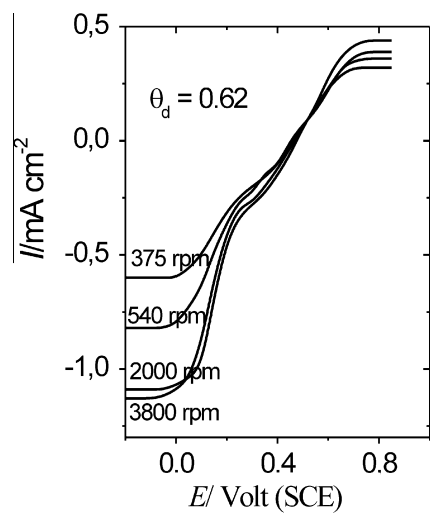

Fig. 2. Steady-state current-potential $(I-E)$ curves for different rotation rates $(\Omega)$ of the rotating disc electrode. A deactivated POAP film $\left(\theta_{\mathrm{d}}=0.62\right) . \Omega$ values are indicated in the figure. POAP film thickness: $60 \mathrm{~nm}$. Electrolyte: $0.1 \mathrm{M} \mathrm{HClO}_{4}+0.4$ $\mathrm{M} \mathrm{NaClO}_{4}+2 \times 10^{-3} \mathrm{M}(\mathrm{HQ} / \mathrm{Q})$

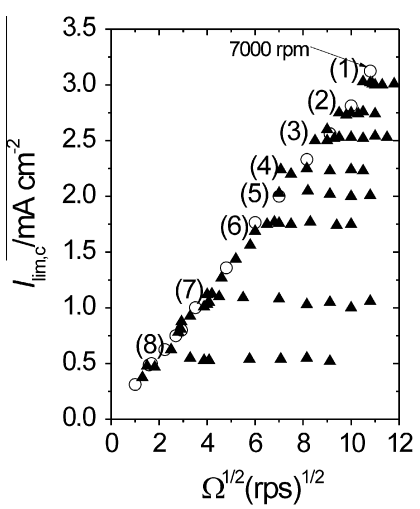

Fig. 3. Levich representations $I_{\text {lim,c }}$ versus $\Omega^{1 / 2}$ for POAP films contacting a $0.1 \mathrm{M}$ $\mathrm{HClO}_{4}+0.4 \mathrm{M} \mathrm{NaClO}_{4}+2 \times 10^{-3} \mathrm{M}(\mathrm{HQ} / \mathrm{Q})$ solution. (O) Empty circles correspond to a nondeactivated POAP film. Black triangles correspond to deactivated films. Numbers from (1) to (8) indicate the different films listed in Table 1.

maximum flux of electrons confined in the polymer, according to Eq. (2) [25]:

$I_{e}=n F A D_{e} c / \phi_{\mathrm{p}}$

In Eq. (2), $c$ is the concentration of redox sites of the polymer and $\phi_{p}$ the polymer film thickness. $D_{\mathrm{e}}$ represents a measure of the electron hopping rate, and $n$ expresses the numbers (fractions) of unit charges per monomer unit of the polymer. $A$ is the electrode area and $F$ the Faraday's constant. Experimental $I_{\mathrm{e}}$ values, corresponding to each one of the eight deactivated POAP films contacting a $2 \times 10^{-3} \mathrm{M} \mathrm{HQ} / \mathrm{Q}$ solution, were extracted from the cathodic plateau and they are listed in Table 2 (3rd column).

As can be seen from Table 2, $I_{\mathrm{e}}$ decreases with increasing $\theta_{\mathrm{d}}$. The constant value of the current $\left(I_{\mathrm{e}}\right)$ at a given $\Omega$ value for deactivated POAP films can be related to a slow electron transport across the POAP film to mediate in the electron-transfer reaction at the polymer|solution interface, as compared with a nondeactivated POAP film. As one increases the flux of $Q$ (increase in $\Omega$ ) from the bulk solution, then if the flux exceeds the supply of electrons from the electrode through the polymer to the electrolyte interface, the rate-limiting step will shift from the limiting transport of $Q$ to the limiting transport of the charge through the polymer. In order to verify this limiting charge-transport process across the POAP film, the $\mathrm{HQ} / \mathrm{Q}$ concentration in solution was varied. It is found that the constant current for a given deactivated film remains unchanged after changing the redox couple concentration in solution [13]. According to Eq. (2), the slower electron transport in deactivated films could be attributed to a decrease of $D_{\mathrm{e}}$. By employing the $I_{\mathrm{e}}$ values listed in Table 2 and using the parameter

Table 2

Efficiency $\left(I_{\mathrm{e}} / I_{0} \times 100\right)$ of a POAP film to act as mediator in the HQ/Q redox reaction after being stored without use in the supporting electrolyte solution for different time periods.

\begin{tabular}{llll}
\hline POAP Film & $\theta_{\mathrm{d}}$ & ${ }^{\mathrm{a}} I_{\mathrm{e}}\left(\mathrm{mAcm}^{-2}\right)$ & ${ }^{\mathrm{b}} I_{\mathrm{e}} / I_{\mathrm{o}} \times 100$ \\
\hline 1 & 0.05 & 3.12 & 97.5 \\
2 & 0.10 & 2.74 & 86 \\
3 & 0.15 & 2.53 & 79 \\
4 & 0.25 & 2.23 & 70 \\
5 & 0.35 & 1.90 & 69 \\
6 & 0.41 & 1.75 & 54 \\
7 & 0.62 & 1.33 & 41 \\
8 & 0.77 & 1.17 & 36
\end{tabular}

${ }^{\text {a }} I_{\mathrm{e}}$ are the different constant current values for the different deactivated films.

b $I_{\mathrm{o}}$ is the current value $\left(3.2 \mathrm{~mA} \mathrm{~cm}^{-2}\right)$ corresponding to the nondeactivated film, which follows the Levich relationship at $\Omega=7000 \mathrm{rpm}$ (Fig. 5). $\left(I_{\mathrm{e}} / I_{\mathrm{o}} \times 100\right)$ represents the efficiency of POAP to act as mediator in the HQ/Q redox reaction. 


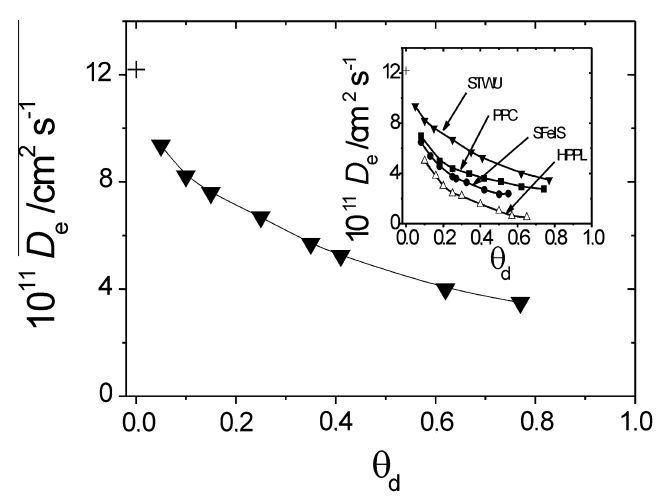

Fig. 4. Electron diffusion coefficient $\left(D_{\mathrm{e}}\right)$ (Eq. (2)) as a function of $\theta_{\mathrm{d}}$ for a POAP film deactivated by STWU. Black triangles correspond to each one of the eight deactivated POAP films listed in Tables 1 and 2. (+) Diffusion coefficient value for a nondeactivated film. $D_{\mathrm{e}}$ values were obtained at the reduced state of POAP, $E=-0.2 \mathrm{~V}$. Electrolyte: $0.1 \mathrm{M} \mathrm{HClO}_{4}+0.4 \mathrm{M} \mathrm{NaClO}_{4}+2 \times 10^{-3} \mathrm{M}(\mathrm{HQ} / \mathrm{Q})$ solution. Inset: Comparison of the electron diffusion coefficient $D_{\mathrm{e}}$ versus $\theta_{\mathrm{d}}$ dependence for different deactivation processes of POAP. Symbols: $(\boldsymbol{\nabla})$ Storage without use; ( $\boldsymbol{\square})$ prolonged potential cycling (values extracted from [11]; $(\bullet)$ interaction with ferric ions $[13,14]$; and $(\Delta)$ high electrode positive potentials [12]).

values $c$ and $n$ reported in [26], $A=1 \mathrm{~cm}^{2}$ and $\phi_{\mathrm{p}}=60 \mathrm{~nm}$ in Eq. (2), one obtains a decrease of $D_{\mathrm{e}}$ from $9.36 \times 10^{-11}$ to $3.50 \times 10^{-11}$ $\mathrm{cm}^{2} \mathrm{~s}^{-1}$ for a relative increase of $\theta_{\mathrm{c}}$ from 0.05 to 0.77 (Fig. 4). The electron diffusion coefficient, $D_{\mathrm{e}}$, in electroactive materials has been expressed in terms of the mean distance between adjacent active redox sites [21], according to $D_{\mathrm{e}}=\left(a^{2} k_{\mathrm{o}}\right)$, where $k_{\mathrm{o}}$ is the intermolecular electron-transfer rate constant and $a$ is the mean distance between two adjacent redox sites. The hopping rate, $k_{0}$, exhibits an exponential dependence on $a$, through the energy $-U(x+a)$ of a state with an electron in the position $x$ along the current direction (see Eq. (23) in Ref. [21]). In this respect, a $k_{\mathrm{o}}$ decrease should be expected as the hopping distance $a$ increases. The decrease of $D_{\mathrm{e}}$ obtained from Eq. (2) could be attributed to an increase in the hopping distance between active redox sites after polymer deactivation by storage. A similar explanation has been given to justify impedance data that show a decrease in the electronic conductivity of POAP with the increase of the solution $p H$ [27]. It has been indicated that POAP deprotonation makes electron hopping difficult decreasing the $k_{\mathrm{o}}$ value.

It is interesting to remark that a constant current $I_{\mathrm{e}}$ independent of $\Omega$ is also obtained for a nondeactivated POAP film in contact with a $0.1 \mathrm{M} \mathrm{HClO}_{4}+0.4 \mathrm{M} \mathrm{NaClO}_{4}+2 \times 10^{-3} \mathrm{M} \mathrm{Q} / \mathrm{HQ}$ solution. However, in this case the constant current is obtained at very high electrode rotation rates $(\Omega>9000 \mathrm{rpm})$. It is possible that at high angular speeds of the rotating disc electrode, the flux into the bulk solution would no longer be laminar, so that the proportionality between the current and $\Omega^{1 / 2}$ should not be expected. However, if the HQ/Q concentration is increased $(0.01 \mathrm{M})$, a constant current $I_{\mathrm{e}}\left(\approx 15 \mathrm{~mA} \mathrm{~cm}^{-2}\right)$ is observed for a nondeactivated film at about $7000 \mathrm{rpm}$ [11]. As this value is reproducible and it decreases as the deactivation of the polymer increases, then our interpretation of a change in the rate-limiting process during the mediated electron-transfer reaction seems to be correct. Similar effects were observed for other polymers [28]. Considering the constant current value obtained for high $\mathrm{HQ} / \mathrm{Q}$ concentrations, a $D_{\mathrm{e}}$ about $1.22 \times 10^{-10} \mathrm{~cm}^{2} \mathrm{~s}^{-1}$ is obtained for a nondeactivated POAP film [11]. This finding seems to indicate that a restriction on electron transport also occurs across a nondeactivated POAP film in contact with a concentrated $\mathrm{HQ} / \mathrm{Q}$ solution and high enough flux into the bulk solution.

On the basis of data shown in Fig. 3, one can assess the efficiency of a POAP film to act as mediator after being stored without use in the supporting electrolyte for different time periods. By taking a high enough electrode rotation rate as reference ( $\Omega=7000 \mathrm{rpm}$, see Levich representation in Fig. 3) to reach a constant current for all the deactivated films treated in the present work, one can calculate the $\left(I_{\mathrm{e}} / I_{\mathrm{o}} \times 100\right)$ ratio, where $I_{\mathrm{e}}$ are the different constant current values for the different deactivated films (see Fig. 3 and Table 2) and $I_{\mathrm{o}}$ is the current value $\left(3.2 \mathrm{~mA} \mathrm{~cm}{ }^{-2}\right.$ at $\Omega=7000 \mathrm{rpm}$ ) corresponding to the nondeactivated film, which follows the Levich relationship at $\Omega=7000 \mathrm{rpm}$. In Table 2, $\left(I_{\mathrm{e}} / I_{\mathrm{o}} \times 100\right)$ represents the efficiency of POAP to act as mediator in the $\mathrm{HQ} / \mathrm{Q}$ redox reaction. The efficiency of a POAP film is reduced to about $36 \%$ for $120 \mathrm{~h}$ STWU.

\section{Surface resistance measurements}

As the $D_{\mathrm{e}}$ decrease with the $\theta_{\mathrm{d}}$ increase obtained from RDEV can be related to an increase in the electron hopping distance between adjacent redox sites and as in previous work it was proved that SR is a useful technique to detect changes in the redox site distribution (mean distance between adjacent redox sites) during the redox switching of a POAP film deposited on a thin gold film [29], some experiments employing the nontraditional SR technique were carried out in this work to study the deactivation of POAP films by STWU. It is interesting to remark that unlike other techniques, such as RDEV and EIS, which are based on complicated models, the SR technique is based on a simple electron dispersion model [30,31]. A review about the application of the surface resistance technique to study different electrochemical processes is given in [16].

Simultaneous voltammetric and SR responses corresponding to a nondeactivated POAP film within the potential range comprised between $-0.2 \mathrm{~V}$ and $0.5 \mathrm{~V}$ are shown in Fig. 6 of Ref. [11]. The SR response corresponding to a nondeactivated POAP film is also shown in Fig. 5 of this work (plot for $\theta_{d}=0$ ). The increase of the gold film resistance during the transition from the reduced state to the oxidized state of a nondeactivated POAP film deposited on it was interpreted on the basis of Eq. (3) [29]:

$\Delta R / R=-3 / 8 G\left(\rho_{\mathrm{m}} l_{\mathrm{m}} / R \phi^{2} \mathrm{~m}\right) \Delta p$

In Eq. (3), $\Delta p=p_{\mathrm{Ox}}-p_{\mathrm{Red}}$ is the difference between the specularity parameters at the oxidized and reduced states of POAP, respectively, $l_{\mathrm{m}}\left(=22 \mathrm{~nm}\right.$ at $\left.25^{\circ} \mathrm{C}\right)$ is the mean free path of the conduction electrons of gold, $\rho_{\mathrm{m}}\left(=2.4 \times 10^{-6} \mathrm{ohm} \mathrm{cm}\right)$ is the bulk resistivity of the massive metal (gold) of the same structure as the metal film, $\phi_{\mathrm{m}}$ is the thickness of the metal film, $G(=2)$ is the relationship between

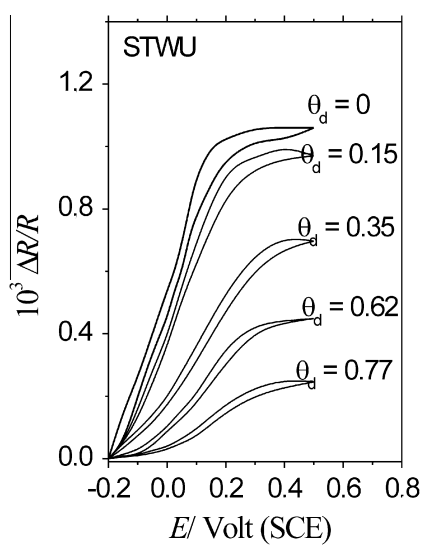

Fig. 5. $\Delta R / R-E$ responses obtained simultaneously with the $(j-E)$ responses shown in Fig. 1. The degrees of deactivation are indicated in the figure. Thickness of the gold film, $\phi_{\mathrm{m}}=30 \mathrm{~nm}$; resistance value of the gold film, $R=20.02 \mathrm{ohm}$. Thickness of the POAP film, $\phi_{\mathrm{P}}=60 \mathrm{~nm}$. Electrolyte: $0.1 \mathrm{M} \mathrm{HClO}_{4}+0.4 \mathrm{M} \mathrm{NaClO}_{4}$. Scan rate: $v=0.01 \mathrm{~V} \mathrm{~s}^{-1}$. 


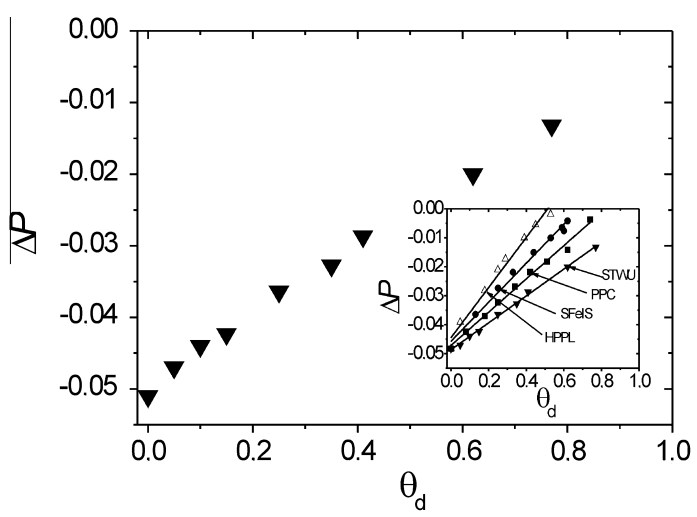

Fig. 6. The $\Delta p$ versus $\theta_{\mathrm{d}}$ dependence in going from the reduced to the oxidized state for a POAP film deactivated by STWU. Thickness of the POAP film, $\phi_{\mathrm{P}}=60 \mathrm{~nm}$. Thickness of the gold film, $\phi_{\mathrm{m}}=30 \mathrm{~nm}$; resistance value of the gold film, $R=20.02 \mathrm{ohm}$. Electrolyte: $0.1 \mathrm{M} \mathrm{HClO}_{4}+0.4 \mathrm{M} \mathrm{NaClO}_{4}$. Scan rate: $v=0.01 \mathrm{~V} \mathrm{~s}^{-1}$ Inset: The $\Delta p$ versus $\theta_{\mathrm{d}}$ dependence in going from the reduced to the oxidized state of POAP for different deactivation processes.

the length $l$ and the width $w$ of the rectangular gold film on which the POAP film was deposited. $R(=20.02 \mathrm{ohm})$ is experimentally measured [16]. The specularity parameter represents the probability of an electron from the interior of the gold film being reflected specularly or diffusely at the gold film surface covered with POAP. The $p$ value ranges from 0 for complete diffuse scattering to 1 for complete specular scattering. The $\Delta R / R$ change can be explained in terms of the generation of electronic entities at the polymer chains near the gold film surface, which occurs by electron transfer across the polymer|gold interface. However, one has to keep in mind that the resistance changes at metal films are not the direct result of the electron transfer between the species on the metal film surface and the metal, but they rather originate from the effect of foreign surface entities on the conduction electrons of the metal itself [16]. Thus, the measured resistance change for a POAP-coated gold film electrode is only related to an interfacial (gold|polymer) electron dispersion process occurring during the reductionoxidation process of the polymer film. In this connection, the redox switching of POAP was interpreted in terms of the oxidation of the amino groups to imine [5]. The increase of $\Delta R / R$ during POAP oxidation (Fig. 5) can be explained in terms of an interfacial distribution of scatterers (imine sites) in the oxidized state with a spacing among them larger than that corresponding to amine sites in the reduced state [16]. That is, the distribution of imine species in the oxidized state of POAP should be less compact than the corresponding distribution of amine species in the reduced state, which should lead to a more diffuse reflection of conduction electrons at the gold|POAP interface (lower specularity parameter at the oxidized state as compared with the value at the reduced one, $p_{\text {ox }}<p_{\text {red }}$ ). Thus, in going from the reduced to the oxidized state of POAP, $\Delta p$ in Eq. (3) is negative. A confirmation that supports a more extended configuration of oxidized sites, as compared with that of reduced ones, at POAP can be given in terms of gaps, which appear during POAP oxidation. In this respect, optical measurements on POAP films reveal that only one in every four or five amine sites is converted to the corresponding imine site [5]. Further confirmation about the different reflecting properties of the oxidized and reduced states of POAP for the gold conduction electrons can be found in the different values of the site interaction parameters $(r)$ obtained from the cathodic and anodic voltammetric response of POAP $[1,2]$. The values of anodic and cathodic site interaction parameters of POAP are: $r_{\mathrm{a}}=-0.55 \mathrm{M}^{-1}$ and $r_{\mathrm{c}}=-0.18 \mathrm{M}^{-1}$, respectively. Both are negative, thus involving a repulsive energy of interaction. As a higher repulsion is observed between oxidized sites than between reduced ones at POAP, a more extended configuration of oxidized sites should be expected as compared with the corresponding distribution of reduced sites.

The POAP film maintains the response shown in Fig. 5 (plot for $\theta_{\mathrm{d}}=0$ ) even after storage without use for a time period of about $31 \mathrm{~h}$. However, after a higher STWU the SR response starts to change. The evolution of the $\Delta R / R$ versus $E$ response for a $60 \mathrm{~nm}$ thick POAP film with the STWU is shown in Fig. 5. Simultaneously with the $\Delta R / R$ versus $E$ response, the evolution of the voltammetric response was also recorded for each POAP film. As the electrode area and the thickness of the POAP films employed in SR were the same as those used in CV and RDEV measurements, the evolution of the voltammetric response with storage was practically the same as that shown in Fig. 1. As can be seen from Fig. 5, the more deactivated the POAP film is, the more attenuated the $\Delta R / R$ change results. As was indicated, the measured resistance change is only related to an interfacial (gold|POAP) electron dispersion process that occurs during reduction-oxidation of the polymer film. This fact was proved by changing the POAP film thickness [22,32]. As was reported in previous papers for other deactivation processes [11], here it was also observed that when the POAP film thickness is increased between $52 \mathrm{~nm}$ and $69 \mathrm{~nm}$ at a constant degree of deactivation, the $\Delta R / R$ change remains practically the same. Obviously, the voltammetric responses depend on the POAP thickness.

Although the absolute values of the specularity parameters at the reduced and oxidized states of POAP at each degree of deactivation are unknown, from the $\Delta R / R$ change and employing Eq. (3), one can obtain the specularity parameter change $\left(\Delta p=p_{\text {Ox }}-p_{\text {Red }}\right)$ at each degree of deactivation. The $\Delta p$ versus $\theta_{\mathrm{d}}$ dependence for a POAP film deactivated by STWU is shown in Fig. 6 . The maximal difference between the reflecting properties at the oxidized $\left(p_{\mathrm{Ox}}\right)$ and reduced $\left(p_{\text {Red }}\right)$ states of POAP for the scattering of gold conduction electrons at the gold/POAP interface occurs for a nondeactivated POAP film $\left(\theta_{\mathrm{d}}=0\right)$. Although $\Delta p$ is always negative, $\Delta p \rightarrow 0$ as the degree of deactivation increases (attenuation of the resistance response). This result could be explained in terms of interfacial distributions of scatterers (redox sites) at the reduced and oxidized states of POAP where the nearest neighbor distance gradually increases, as the degree of deactivation increases, until their reflecting properties for the conduction electrons match one another at a high enough $\theta_{\mathrm{d}}$ value. The existence of a more spread redox site distribution at the reduced state of POAP as the degree of deactivation increases seems to be supported by the decrease of the electron diffusion coefficient value with the increase in $\theta_{d}$ obtained from steady-state RDEV measurements (Fig. 4). As was indicated, this effect can be explained in terms of an increase of the electron hopping distance in the bulk redox site distribution that reduces the electron-transport rate inside the polymer matrix. Unfortunately, RDEV measurements at the anodic plateau of POAP show that the anodic limiting current $(E>0.8 \mathrm{~V})$ corresponds to the oxidation of $H Q$ species that penetrate through the polymer film to reach the metal surface, and then, the dependence of the electron diffusion coefficient at the oxidized state of POAP on the degree of deactivation cannot be obtained.

At this point it should be remarked that the resistance increase $(\Delta R / R)$ of a gold film coated with POAP, as the polymer goes from the reduced $(E=-0.2 \mathrm{~V})$ to the oxidized state $(E=0.4 \mathrm{~V})$, is related to the variation in the type and distribution of scatterers (amine and imine species) at the $\mathrm{POAP} /$ gold interface for the conduction electrons of the gold film. In this regard, surface resistance only refers to the dispersion of conduction electrons of the metal film, but it is not, at least directly, related to the polymer conduction itself. With regard to POAP conduction, the probable redox reaction of POAP is shown in Fig. 4 of Ref. [27]. While both the fully reduced state and the fully oxidized state of POAP have no radical species, 
and then, they can only present very low electronic conductivities, the half-oxidized state is a radical species that should exhibit the maximum electronic conductivity. The prediction of the existence of a conductive species in the partially oxidized state of POAP is also verified from the optical measurements reported in [5], where it is demonstrated that potential cycling while holding the wavelength at $750 \mathrm{~nm}$ yields a transient response in the absorbance peaking at the peak potential of the voltammetric response ( $E=0.05 \mathrm{~V}$ vs. SCE). Impedance measurements also show a maximum conductivity value for POAP $\left(4.55 \pm 0.2 \times 10^{-7} \mathrm{~S} \mathrm{~cm}^{-1}\right)$ around $0.05 \mathrm{~V}$ (versus SCE) at $p H 1$ [26].

\section{Comparison of the effect of different deactivation procedures on the electron transport process inside a POAP film and electron scattering at the gold/POAP interface}

At this point, the effects of the STWU on both the bulk electron transport rate across a POAP film and the interfacial electron scattering at the gold/POAP interface are compared with the effects caused by other deactivation methods employed in previous work [11-14]. The effect of deactivation on the bulk charge-transport process of POAP films in previous work [11-14] was also studied employing RDEV, and experimental data were interpreted on the basis of the electron hopping model. The comparison of the $D_{\mathrm{e}}$ versus $\theta_{\mathrm{d}}$ dependence obtained in this work for POAP films deactivated by STWU with those obtained in previous work employing HPPL, PPC and SFeIS is shown in the inset of Fig. 4. High positive potential limits $(E>0.5 \mathrm{~V})$ seem to cause the strongest decrease in the electron transport rate at the reduced state of POAP. The effects caused by PPC and SFeIS on the electron conduction seem to be intermediate between those caused by HPPL and STWU. It is observed from the inset in Fig. 4 that as $\theta_{\mathrm{d}} \rightarrow 0$, all $D_{\mathrm{e}}$ versus $\theta_{\mathrm{d}}$ dependences seem to tend toward the $D_{\mathrm{e}}$ value corresponding to a nondeactivated film. Also, at a constant $\theta_{\mathrm{d}}$ value, the electron diffusion coefficient follows the order $D_{\mathrm{e}}(\mathrm{STWU})>D_{\mathrm{e}}(\mathrm{PPC})>$ $D_{\mathrm{e}}(\mathrm{SFeIS})>D_{\mathrm{e}}(\mathrm{HPPL})$. In this regard, although a given degree of deactivation can be reached employing different deactivation processes, the effect on the bulk electron conduction depends on the deactivation procedure employed to achieve such degree of deactivation. Then, the sequence of $D_{\mathrm{e}}$ values at a constant $\theta_{\mathrm{d}}$ value could be explained in terms of the electron hopping model by assuming that at each degree of deactivation, redox site configurations with different mean distances between redox sites are possible. In this regard, at a constant degree of deactivation, the distribution of active redox sites of a POAP film subjected to HPPL should be more spread (higher mean distance between two adjacent redox sites) than the distribution of active redox sites achieved after deactivation of the electrode employing any of the other procedures.

The SR was also employed in previous work to investigate changes in the electronic properties at the gold/POAP interface during the deactivation of POAP [11-14]. The resistance response of POAP films deactivated by PPC was studied in [11]. As for a POAP film deactivated by STWU, an attenuation of the $\Delta R / R$ change in going from the reduced to the oxidized state of POAP is observed as the degree of deactivation increases. The higher the deactivation degree is, the more pronounced the $\Delta R / R$ attenuation becomes (see Fig. 7 in [11]).

The SR response of POAP films deactivated by HPPL and that of POAP films deactivated by SFeIS are shown in [12] and [14], respectively. However, in both papers, the $\Delta R / R$ change was recorded taking the oxidized state of POAP as reference. Then, for comparison purposes, resistance measurements for POAP films deactivated by employing HPPL and SFeIS were repeated in the present work, and the resistance responses for different deactivated films were compensated at the reduced state of POAP $(E=-0.2 \mathrm{~V})$. Figs. 7

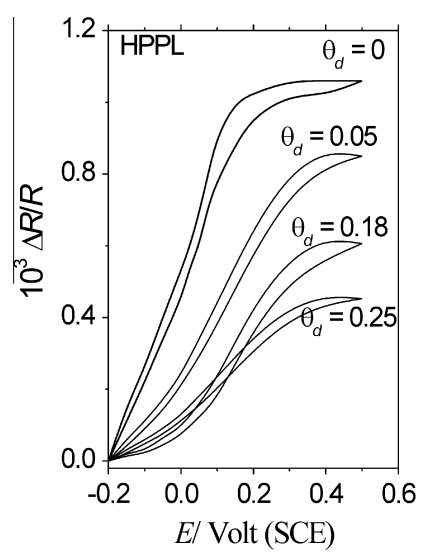

Fig. 7. $\Delta R / R-E$ responses of a POAP film gradually deactivated by HPPL. Thickness of the gold film, $\phi_{\mathrm{m}}=30 \mathrm{~nm}$; resistance value of the gold film, $R=20.02 \mathrm{ohm}$. Thickness of the POAP film, $\phi_{\mathrm{P}}=60 \mathrm{~nm}$. Electrolyte: $0.1 \mathrm{M} \mathrm{HClO}_{4}+0.4 \mathrm{M} \mathrm{NaClO}_{4}$. Scan rate: $v=0.01 \mathrm{~V} \mathrm{~s}^{-1}[12]$.

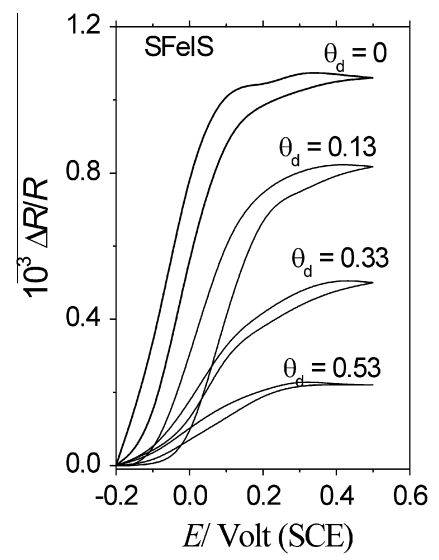

Fig. 8. $\Delta R / R-E$ response of a POAP film gradually deactivated by SFeIS. Thickness of the gold film, $\phi_{\mathrm{m}}=30 \mathrm{~nm}$; resistance value of the gold film, $R=20.02 \mathrm{ohm}$. Thickness of the POAP film, $\phi_{\mathrm{P}}=60 \mathrm{~nm}$. Electrolyte: $0.1 \mathrm{M} \mathrm{HClO}_{4}+0.4 \mathrm{M} \mathrm{NaClO}_{4}$. Scan rate: $v=0.01 \mathrm{~V} \mathrm{~s}^{-1}[13,14]$.

and 8 show the resistance change in going from the reduced to the oxidized state of POAP films subjected to HPPL and deactivated by SFeIS, respectively. As the magnitude of the resistance change is independent of the reference state selected to start the electrode potential scan, the resistance responses shown in Figs. 7 and 8 and those shown in [12] and [14] are, respectively, the same at the corresponding degree of deactivation. As can be seen from Figs. 7 and 8, a gradual attenuation of the resistance response is always observed as the film becomes more deactivated.

As previously indicated, by employing Eq. (3), one can compare the specularity parameter change $\left(\Delta p=p_{\mathrm{Ox}}-p_{\text {Red }}\right)$ in going from the reduced to the oxidized state of POAP at each degree of deactivation for the different deactivation processes. The $\Delta p$ versus $\theta_{\mathrm{d}}$ dependences for the different deactivation processes are shown in the inset of Fig. 6. $\Delta p \rightarrow 0$ at different rates for the different deactivation processes: $\Delta p / \theta_{\mathrm{d}} \quad(\mathrm{HPPL})>\Delta p / \theta_{\mathrm{d}} \quad(\mathrm{SFeIS})>\Delta p / \theta_{\mathrm{d}}$ $(\mathrm{PPC})>\Delta p / \theta_{\mathrm{d}}$ (STWU). This fact should be indicative of the existence of scatterer interfacial distributions for the dispersion of gold conduction electrons, at the reduced and oxidized states of POAP, where the nearest neighbor distance between them (redox sites) at constant degree of deactivation is different according to the method employed to deactivate the polymer film. In this regard, the $\Delta p$ value closer to 0 at each degree of deactivation for a POAP film subjected to HPPL should indicate the existence of redox site 
interfacial distributions with the highest mean distance between adjacent redox sites as compared with those obtained from films deactivated by any of the other procedures. This finding seems to be consistent with the $D_{\mathrm{e}}$ versus $\theta_{\mathrm{d}}$ dependence for the reduced state of POAP shown in the inset of Fig. 4, where it is observed that a POAP film deactivated by HPPL, as compared with films deactivated by any of the other procedures, exhibits the most spread bulk redox site configuration at each degree of deactivation. Then, by comparing the insets in Figs. 4 and 6 it is possible to conclude that although a given degree of deactivation of POAP can be reached employing different procedures, the effects on the electron-transport process inside the polymer and the surface scattering of the metal conduction electrons at the metal/polymer interface depend not only on the number of active redox sites but also on their distribution.

The deactivation of POAP films by STWU was also studied employing EIS. This technique allows one to obtain a complete series of charge-transport and charge-transfer parameters of a polymer film, such as diffusion coefficients for electron $\left(D_{\mathrm{e}}\right)$ and ion $\left(D_{\mathrm{i}}\right)$ transport and interfacial resistances related to ion $\left(R_{\mathrm{i}}^{\mathrm{f} \mid \mathrm{s}}\right)$ and electron $\left(R_{\mathrm{m} \mid \mathrm{f}}, R_{\mathrm{e}}^{\mathrm{f} \mid \mathrm{s}}\right)$ transfer across the polymer/solution and metal/polymer interfaces, respectively. EIS results extracted from POAP films deactivated by STWU and their comparison with those obtained for films deactivated by prolonged potential cycling (PPC), high positive potential limits (HPPL) and soaking in a ferric ion solution (SFeIS) will be described in a forthcoming paper.

\section{Conclusions}

Poly(o-aminophenol) film electrodes maintain their conducting properties unaltered after being stored without use in a deoxygenated supporting electrolyte solution for about $31 \mathrm{~h}$. However, a loss of electroactivity is observed as the storage time without use is extended beyond $32 \mathrm{~h}$. In this regard, a gradual attenuation of the voltammetric response of POAP is observed with the increase in the storage time without use. A decrease of the electron diffusion coefficient value with the increase in the degree of deactivation is also observed from RDEV measurements. The slower electron transport with the increase in the degree of deactivation was attributed to the increase of the electron hopping distance between redox sites. The relative surface resistance change of a gold film coated with poly(o-aminophenol) is also gradually attenuated by the increase in the storage time without use. This attenuation was attributed to interfacial distributions of scatterers (redox sites at the gold/polymer interface) at the reduced and oxidized states of POAP where the nearest neighbor distance gradually increases, as the degree of deactivation increases, until their reflecting properties for the conduction electrons match one another at a high enough degree of deactivation value. This explanation seems to be consistent with the increase in the electron hopping distance between redox sites, proposed to explain the decrease of the diffusion coefficient with the increase in the degree of deactivation obtained from RDEV.

The deactivation of poly (o-aminophenol) films by storage time without use was compared with other deactivation procedures reported in previous work: prolonged potential cycling (PPC), application of high positive potential limits (HPPL) and soaking in a ferric ion solution (SFeIS). Steady-state RDEV measurements show that at each degree of deactivation, the bulk electron transport rate at the reduced state of POAP follows the sequence: $D_{\mathrm{e}}(\mathrm{STWU})>D_{\mathrm{e}}(\mathrm{PPC})>D_{\mathrm{e}}$ (SFeIS $)>D_{\mathrm{e}}(\mathrm{HPPL})$. As a $D$ e decrease can be associated with an increase in the electron hopping distance between redox sites, it can be concluded that the most spread redox site distribution is obtained for a POAP film deactivated by applying HPPL as compared with a film deactivated by any of the other deactivation procedures. Potentiodynamic SR measurements seem to indicate for all deactivation procedures that interfacial redox site distributions at the reduced and oxidized states of POAP become less compact and tend to match one another as the degree of deactivation of the polymer increases. However, the rate of change depends on the deactivation procedure employed. Considering the four deactivation methods, the highest rate of change is observed for POAP films deactivated by applying HPPL. Then, a POAP film deposited on a gold film electrode subjected to HPPL exhibits the most spread redox site configuration (lower bulk electron transport rate and more diffuse scattering at the metal/polymer interface for the metal conduction electrons) as compared with a POAP film deactivated by any of the other deactivation methods.

\section{Conflict of interest}

There is no conflict of interest among authors.

\section{Acknowledgements}

The author gratefully acknowledges the Consejo Nacional de Investigaciones Científicas y Técnicas (CONICET) and also the Facultad de Ciencias Exactas, National University of La Plata (UNLP).

\section{References}

[1] C. Barbero, J.J. Silber, L. Sereno, J. Electroanal. Chem. 291 (1990) 81-101.

[2] C. Barbero, J.J. Silber, L. Sereno, J. Electroanal. Chem. 263 (1989) 333-352.

[3] T. Ohsaka, S. Kunimura, N. Oyama, Electrochim. Acta 33 (1988) 639-645.

[4] R. Tucceri, P.M. Arnal, A.N. Scian, Can. J. Chem. 91 (2013) 91-112.

[5] R.I. Tucceri, C. Barbero, J.J. Silber, L. Sereno, D. Posadas, Electrochim. Acta 42 1997) 919-927.

[6] O. Levin, V. Kondratiev, V. Malev, Electrochim. Acta 50 (2005) 1573-1585.

[7] M.C. Miras, A. Badano, M.M. Bruno, C. Barbero, Portugaliae Electrochimica Acta 21 (2003) 235-243.

[8] J. Yano, H. Kawakami, S. Yamasaki, Y. Kanno, J. Electrochem. Soc. 148 (2001) E61-E65.

[9] M.A. Valdés García, P. Tuñón Blanco, A. Ivaska, Electrochim. Acta 43 (1998) 3533-3539.

[10] S.M. Golabi, A. Nozad, Electroanalysis 15 (2003) 278-286.

[11] R. Tucceri, J. Electroanal. Chem. 717-718 (2014) 131-139.

[12] R. Tucceri, J. New Mater. Electrochem. Syst. 8 (2005) 305-315.

[13] R. Tucceri, J. Electroanal. Chem. 633 (2009) 198-206.

[14] R. Tucceri, J. Electroanal. Chem. 659 (2011) 83-91.

[15] C. Barbero, J. Zerbino, L. Sereno, D. Posadas, Electrochim. Acta 32 (1987) 693697.

[16] R. Tucceri, Surf. Sci. Rep. 56 (2004) 85-157.

[17] R.I. Tucceri, D. Posadas, J. Electrochem. Soc. 130 (1983) 104-107.

[18] R.I. Tucceri, D. Posadas, J. Electrochem. Soc. 128 (1981) 1478-1483.

[19] J.O. Chambers, G. Inzelt, Anal. Chem. 57 (1985) 1117-1121.

[20] P. Daum, R.W. Murray, J. Phys. Chem. 85 (1981) 389-396.

[21] Ch.E.D. Chidsey, R.W. Murray, J. Phys. Chem. 90 (1986) 1479-1484.

[22] A. Bonfranceschi, A. Pérez Córdoba, S. Keunchkarian, S. Zapata, R. Tucceri, J. Electroanal. Chem. 477 (1999) 1.

[23] E. Laviron, J. Electroanal. Chem. 112 (1980) 1-9.

[24] C.P. Andrieux, J.M. Savéant, J. Electroanal. Chem. 111 (1980) 377-381.

[25] C. Deslouis, B. Tribollet, in: H. Gerischer, C. Tobias (Eds.), Advances in Electrochemical Science and Engineering, vol. 2, VCH Publishers, New York, USA, 1992, p. 205.

[26] C. Barbero, R.I. Tucceri, D. Posadas, J.J. Silber, L. Sereno, Electrochim. Acta 40 (1995) 1037-1040.

[27] T. Komura, Y. Ito, T. Yamaguti, K. Takahasi, Electrochim. Acta 43 (1998) 723731.

[28] W.J. Albery, M.G. Boutelle, A.R. Hillman, J. Electroanal. Chem. 182 (1985) 99111.

[29] R. Tucceri, J. Electroanal. Chem. 562 (2004) 173-186.

[30] F. Fuchs, Proc. Camb. Phyl. Soc. Math. Phys. Sci 34 (1938) 100-176.

[31] E.H. Sondheimer, Adv. Phys. 1 (1952) 1.

[32] M. Ortega, Thin Solid Films 371 (2000) 28. 